VoL. 44 (1991) [177-188]

\title{
RATE OF APPROXIMATION OF FUNCTIONS OF BOUNDED VARIATION BY MODIFIED LUPAS OPERATORS
}

\author{
Wang Yuankwei and Guo Shunsheng
}

This paper discusses the rate of approximation of functions of bounded variation using the Modified Lupas operator. We obtain an approximation theorem and our estimate is essentially the best possible.

\section{INTRODUCTION}

Let $f$ be a function defined on $(0, \infty)$ with bounded variation in each finite interval and $f(x)=O\left(x^{r}\right) x \rightarrow \infty$. We denote $\{f\}$ by $B V_{\text {loc, },}(0, \infty)$. The Modified Lupas operator $M_{n}$ applied to $f$ is

where

$$
\begin{gathered}
M_{n}(f, x)=(n-1) \sum_{k=0}^{\infty} P_{n k}(x) \int_{0}^{\infty} P_{n k}(t) f(t) d t \\
P_{n k}(t)=\left(\begin{array}{c}
n+k-1 \\
k
\end{array}\right) \frac{t^{k}}{(1+t)^{n+k}} .
\end{gathered}
$$

It is also written as

$$
\begin{gathered}
\int_{0}^{\infty} H_{n}(x, t) f(t) d t \\
H_{n}(x, t)=(n-1) \sum_{k=0}^{\infty} P_{n k}(x) P_{n k}(t) .
\end{gathered}
$$

where

This kernel is positive and

$$
\int_{0}^{\infty} H_{n}(x, t) d t=1
$$

therefore $M_{n}(f, x)$ is linear positive [1].

In this paper, the main result is the following:

Received 5th September 1990

Copyright Clearance Centre, Inc. Serial-fee code: 0004-9729/91 \$A2.00+0.00. 
THEOREM. Let $f$ be a function belonging to $B V_{\text {loc, } r}(0, \infty)$. Then for every $x \in(0, \infty)$ and $n$ sufficiently large, we have

$$
\begin{aligned}
& \left|M_{n}(f, x)-\frac{1}{2}(f(x+)-f(x-))\right| \leqslant \frac{5(1+x)}{n x} \sum_{k=1}^{n} \bigvee_{x-\frac{x}{\sqrt{k}}}^{x+\frac{x}{\sqrt{k}}}\left(g_{x}\right) \\
& \quad+\frac{50}{\sqrt{n}}\left(\frac{1+x}{x}\right)^{3 / 2}|f(x+)-f(x-)|+\frac{(1+x)^{r}}{x^{4}} O\left(\frac{1}{n^{2}}\right)
\end{aligned}
$$

where

$$
g_{x}(t)= \begin{cases}f(t)-f(x+) & x<t<\infty \\ 0 & t=x \\ f(t)-f(x-) & 0 \leqslant t<x\end{cases}
$$

The proof of the theorem will use the Bojanic method [2] and some results of probability theory.

\section{Lemmas}

LEMmA 1. If $\left\{\xi_{k}\right\}(k \geqslant 1)$ are independent random variables with the same distribution functions and $0<D \xi_{k}<\infty, \beta_{3}=E\left(\xi_{r}-\xi_{i}\right)^{3}<\infty$, then

$$
\max _{y}\left|P\left(\frac{1}{b_{1} \sqrt{n}} \sum_{k=1}^{n}\left(\xi_{k}-a_{1}\right) \leqslant y\right)-\frac{1}{\sqrt{2 \pi}} \int_{-\infty}^{y} e^{-t^{2} / 2} d t\right|<\frac{c}{\sqrt{n}} \frac{\beta_{3}}{b_{1}^{3}}
$$

where $a_{1}=E\left(\xi_{1}\right)$ (expectation of $\left.\xi_{1}\right), b_{1}^{2}=D \xi_{1}=E\left(\xi_{1}-E \xi_{1}\right)^{2}$ and $1 / \sqrt{2 \pi} \leqslant c<$ 0.82 (see [3]).

LEMMA 2. [3] If $\left\{\xi_{i}\right\}$ are independent random variables with the same geometric distribution functions $P\left(\xi_{i}=k\right)=x^{k}(1-x), i=1,2, \ldots$, then $E \xi_{i}=x /(1-x)$, $D \xi_{i}=x /(1-x)^{2}, \eta_{n}=\sum_{i=1}^{n} \xi_{i}$ with

$$
P\left(\eta_{n}=k\right)=\left(\begin{array}{c}
n+k-1 \\
k
\end{array}\right) x^{k}(1-x)^{n} .
$$

LEMma 3. For every $x \in(0,+\infty), k \in \mathbb{N}$, we have

$$
P_{n k}(x) \leqslant \frac{33}{\sqrt{n}}\left(\frac{1+x}{x}\right)^{3 / 2}
$$

Proof: Since

$$
P_{n k}(x)=\left(\begin{array}{c}
n+k-1 \\
k
\end{array}\right) \frac{x^{k}}{(1+x)^{n+k}} \quad x \in(0, \infty),
$$


we take $t=x /(1+x) ;$ then $t \in(0,1)$ and

$$
P_{n k}(x)=\left(\begin{array}{c}
n+k-1 \\
k
\end{array}\right) t^{k}(1-t)^{n}
$$

Using Lemma 2 we have

$$
\left(\begin{array}{c}
n+k-1 \\
k
\end{array}\right) t^{k}(1-t)^{n}=P\left(k-1<\eta_{n} \leqslant k\right)=P\left(w(k-1, n)<w\left(\eta_{n}, n\right) \leqslant w(k, n)\right)
$$

where $w(k, n)=(x(1-t)-n t) / \sqrt{n t}$. Using Lemma 1 we have $a_{1}=t /(1-t), b_{1}=$ $\sqrt{t} /(1-t)$. Hence

$$
\left|\left(\begin{array}{c}
n+k-1 \\
k
\end{array}\right) t^{k}(1-t)^{n}-\frac{1}{\sqrt{2 \pi}} \int_{I} e^{-t^{2} / 2} d t\right|<2 \frac{\beta_{3}}{\sqrt{n}(\sqrt{t} /(1-t))^{3}}
$$

where

$$
\begin{aligned}
I & =[w(k-1, n), w(k, n)], \\
\beta_{3} & =E\left(\xi_{k}-\frac{t}{1-t}\right)^{3}=\sum_{k=0}^{\infty}\left(k-\frac{t}{1-t}\right)^{3} t^{k}(1-t) \\
& \leqslant \sum_{k=0}^{\infty}\left[k^{3}+3 k^{2} \frac{t}{1-t}+3 k\left(\frac{t}{1-t}\right)^{2}+\left(\frac{t}{1-t}\right)^{3}\right] t^{k}(1-t) .
\end{aligned}
$$

Since

$$
\begin{aligned}
\sum_{k=0}^{\infty} t^{k}(1-t) & =1, & & \sum_{k=0}^{\infty} k t^{k}(1-t)=\frac{t}{1-t}, \\
\sum_{k=0}^{\infty} k^{2} t^{k}(1-t) & =\frac{t(1+t)}{1-t^{2}}, & & \sum_{k=0}^{\infty} k^{3} t^{k}(1-t)=\frac{t^{3}+4 t^{2}+t}{(1-t)^{3}}
\end{aligned}
$$

therefore $\beta_{3} \leqslant 16 /(1-t)^{3}$.

But the second term on the left side of $(2.5)$ is not greater than $(1-t) / \sqrt{2 \pi n t}$. Hence we have

$$
\left(\begin{array}{c}
n+k-1 \\
k
\end{array}\right) t^{k}(1-t)^{n} \leqslant \frac{33}{\sqrt{n} t^{3 / 2}}
$$

therefore

$$
P_{n k}(x) \leqslant \frac{33}{\sqrt{n}}\left(\frac{1+x}{x}\right)^{3 / 2}, \quad \text { and }(2.3) \text { is proved. }
$$

LEMMA 4. For every $k \geqslant 0$ we have

$$
\sum_{j=0}^{k} P_{n-1, j}(x)=(n-1) \int_{x}^{\infty} P_{n k}(t) d t
$$


Proof: This can easily be proved by differentiating both the left-hand and righthand sides.

LEMMA 5. If $n$ is sufficiently large, for every $k \geqslant 0$ we have

$$
\left|\sum_{j=0}^{k} P_{n-1, j}(x)-\sum_{j=0}^{k} P_{n j}(x)\right| \leqslant \frac{33}{\sqrt{n}}\left(\frac{1+x}{x}\right)^{3 / 2} .
$$

Proof: From (2.2) we have $\sum_{j=0}^{k} P_{n j}(x)=P\left(\eta_{n} \leqslant k\right)$. Again using Lemma 1 we have

$$
\left|\sum_{j=0}^{k} P_{n j}(x)-\frac{1}{\sqrt{2 \pi}} \int_{-\infty}^{w(k, n)} e^{-u^{2} / 2} d u\right|<\frac{16}{\sqrt{n}}\left(\frac{1+x}{x}\right)^{3 / 2}
$$

hence

$$
\begin{aligned}
& \left|\sum_{j=0}^{k} P_{n-1, j}(x)-\sum_{j=0}^{k} P_{n j}(x)\right| \\
& \quad<\left|\frac{1}{\sqrt{2 \pi}} \int_{w(k, n)}^{w(k, n-1)} e^{-u^{2} / 2} d u\right|+\frac{32}{\sqrt{n}}\left(\frac{1+x}{x}\right)^{3 / 2} \leqslant \frac{33}{\sqrt{n}}\left(\frac{1+x}{x}\right)^{3 / 2} .
\end{aligned}
$$

LEMMA 6. If $n$ is sufficiently large and $x \in(0, \infty)$, then

$$
\begin{aligned}
& \frac{x(1+x)}{n} \leqslant M_{n}\left((t-x)^{2}, x\right) \leqslant \frac{3 x(1+x)}{n} \\
& M_{n}\left((t-x)^{4}, x\right)=O\left(\frac{1}{n^{2}}\right) .
\end{aligned}
$$

Proof: Let

$$
T_{n m}=(n-r+1) \sum_{k=0}^{\infty} P_{n+r, k}(x) \int_{0}^{\infty} P_{n-r, k+r}(t)(t-x)^{m} d t .
$$

Then

$$
\begin{aligned}
T_{n 2} & =(n-r+1) \sum_{k=0}^{\infty} P_{n+r, k}(x) \int_{0}^{\infty} P_{n+r, k}(x) \int_{0}^{\infty} P_{n-r, k+r}(t)(t-x)^{2} d t \\
& =\frac{2(n-1) x(1+x)}{(n-r-2)(n-r-3)}+\frac{(r+1)(r+2)(1+2 x)^{2}}{(n-r-2)(n-r-3)} .
\end{aligned}
$$


Putting $r=0$ we have

$$
\begin{aligned}
T_{n m} & =(n+1) \sum_{k=0}^{\infty} P_{n k}(x) \int_{0}^{\infty} P_{n k}(t)(t-x)^{m} d t=O\left(\frac{1}{n^{[(m+1) / 2]}}\right) \\
T_{n 2} & =\frac{2(n-1) x(1+x)}{(n-2)(n-3)}+\frac{2(1+2 x)^{2}}{(n-2)(n-3)}
\end{aligned}
$$

From this, (2.8) and (2.9) can be proved (see [4]).

LEMMA 7. In $n$ is sufficiently large and $x \in(0, \infty)$, then for $0 \leqslant y<x$, we have

$$
\int_{0}^{y} H_{n}(x, t) d t \leqslant \frac{3 x(1+x)}{n(x-y)^{2}}
$$

for $x<z<\infty$, we have

$$
\int_{z}^{\infty} H_{n}(x, t) d t \leqslant \frac{3 x(1+x)}{n(z-x)^{2}}
$$

Proof: Since $0 \leqslant y<x$, for $t \in[0, y]$ we have $(x-t) /(x-y) \geqslant 1$.

From

$$
M_{n}(f, x)=\int_{0}^{\infty} H_{n}(x, t) f(t) d t
$$

we have

$$
\frac{x(1+x)}{n} \leqslant \int_{0}^{\infty} H_{n}(x, t)(t-x)^{2} d t \leqslant \frac{3 x(1+x)}{n}
$$

therefore

$$
\begin{aligned}
& \int_{0}^{y} H_{n}(x, t) d t \leqslant \int_{0}^{y}\left(\frac{x-t}{x-y}\right)^{2} H_{n}(x, t) d t \\
& \leqslant \frac{1}{(x-y)^{2}} \int_{0}^{\infty}(x-t)^{2} H_{n}(x, t) d t \leqslant \frac{3 x(1+x)}{n(x-y)^{2}}
\end{aligned}
$$

proving (2.12). The proof of (2.13) is similar.

\section{Proof of the Theorem}

Now

$$
f(t)=\frac{f(x+)+f(x-)}{2}+g_{x}(t)+\frac{f(x+)-f(x-)}{2} \operatorname{sign}(t-x)
$$

hence

$$
\begin{aligned}
& \left|M_{n}(f, x)-\frac{1}{2}(f(x+)+f(x-))\right| \\
& \quad \leqslant\left|M_{n}\left(g_{x}, x\right)\right|+\frac{1}{2}|f(x+)-f(x-)|\left|M_{n}(\operatorname{sign}(t-x), x)\right| .
\end{aligned}
$$


Thus to estimate $\left|M_{n}(f, x)-(f(x+)-f(x-)) / 2\right|$ we need an estimate for $M_{n}\left(g_{x}, x\right)$ and $M_{n}(\operatorname{sign}(t-x), x)$.

To estimate $M_{n}(\operatorname{sign}(t-x), x)$, we first decompose it into two parts as follows:

$$
\begin{aligned}
M_{n}(\operatorname{sign}(t-x), x)= & \int_{0}^{\infty} \operatorname{sign}(t-x) H_{n}(x, t) d t=\int_{x}^{\infty} H_{n}(x, t) d t \\
& -\int_{0}^{x} H_{n}(x, t) d t \stackrel{\text { def }}{=} A_{n}(x)-B_{n}(x) .
\end{aligned}
$$

Using Lemma 4, we have

$$
\begin{aligned}
A_{n}(x) & =\int_{x}^{\infty} H_{n}(x, t) d t=\int_{x}^{\infty}(n-1) \sum_{k=0}^{\infty} P_{n k}(x) P_{n k}(t) d t \\
& =(n-1) \sum_{k=0}^{\infty} P_{n k}(x) \int_{x}^{\infty} P_{n k}(t) d t \\
& =\sum_{k=0}^{\infty} P_{n k}(x) \sum_{j=0}^{k} P_{n-1, j}(x) .
\end{aligned}
$$

By Lemma 5, it follows that

$$
\begin{aligned}
& \left|A_{n}(x)-\sum_{k=0}^{\infty} P_{n k}(x) \sum_{j=0}^{k} P_{n j}(x)\right| \\
& =\left|\sum_{k=0}^{\infty} P_{n k}(x) \sum_{j=0}^{k} P_{n j}(x)-\sum_{k=0}^{\infty} P_{n k}(x) \sum_{j=0}^{k} P_{n-1, j}(x)\right| \\
& \quad \leqslant \sum_{k=0}^{\infty} P_{n k}(x) \frac{33}{\sqrt{n}}\left(\frac{1+x}{x}\right)^{3 / 2} \leqslant \frac{33}{\sqrt{n}}\left(\frac{1+x}{x}\right)^{3 / 2} .
\end{aligned}
$$

Let

$$
\begin{aligned}
S & =\sum_{k=0}^{\infty}\left(P_{n k}(x) \sum_{j=0}^{k} P_{n j}(x)\right) \\
& =P_{n 0} P_{n 0}+P_{n 1}\left(P_{n 0}+P_{n 1}\right)+\cdots+P_{n n}\left(P_{n 0}+\cdots+P_{n n}\right)+\cdots
\end{aligned}
$$

Since

$$
\begin{aligned}
1 & =\left(P_{n 0}+P_{n 1}+\cdots+P_{n m}+\cdots\right)\left(P_{n 0}+P_{n 1}+\cdots+P_{n m}+\cdots\right) \\
& =P_{n 0}\left(P_{n 0}+P_{n 1}+\cdots+P_{n m}+\cdots\right)+P_{n 1}\left(P_{n 0}+\cdots\right)+P_{n m}\left(P_{n 0}+\cdots\right)+\cdots
\end{aligned}
$$


we have

$$
\begin{gathered}
1-S=P_{n 0}\left(P_{n 1}+P_{n 2}+\cdots\right)+P_{n 1}\left(P_{n 2}+P_{n s}+\cdots\right) \\
\quad+P_{n m}\left(P_{n, m+1}+P_{n, m+2}+\cdots\right)+\cdots \\
=P_{n 1} P_{n 0}+P_{n 2}\left(P_{n 0}+P_{n 1}\right)+\cdots \\
\quad+P_{n m}\left(P_{n 0}+P_{n 1}+\cdots+P_{n, m-1}\right)+\cdots, \\
2 S-1=P_{n 0}^{2}+P_{n 1}^{2}+\cdots+P_{n m}^{2}+\cdots .
\end{gathered}
$$

and

Using Lemma 3, we obtain

$$
\begin{aligned}
\left|S-\frac{1}{2}\right| & =\frac{1}{2}\left(P_{n 0}^{2}+P_{n 1}^{2}+\cdots\right)+\frac{1}{2} \sum_{k=0}^{\infty} P_{n k}^{2} \\
& \leqslant \frac{1}{2} \sum_{k=0}^{\infty} P_{n k}\left[\frac{33}{\sqrt{n}}\left(\frac{1+x}{x}\right)^{3 / 2}\right] \leqslant \frac{33}{2 \sqrt{n}}\left(\frac{1+x}{x}\right)^{3 / 2} .
\end{aligned}
$$

By (3.2), (3.3) and $B_{n}(x)=1-A_{n}(x)$, we have

$$
\left|A_{n}(x)-B_{n}(x)\right|=\left|2 A_{n}(x)-1\right| \leqslant \frac{100}{\sqrt{n}}\left(\frac{1+x}{x}\right)^{3 / 2} .
$$

Hence

$$
\left|M_{n}(\operatorname{sign}(t-x), x)\right| \leqslant \frac{100}{\sqrt{n}}\left(\frac{1+x}{x}\right)^{3 / 2} .
$$

The estimate of $M_{n}\left(g_{x}, x\right)$ is similar to [4]. We first decompose $[0,+\infty)$ into three parts, as follows:

$$
I_{1}=\left[0, x-\frac{x}{\sqrt{n}}\right], \quad I_{2}=\left[x-\frac{x}{\sqrt{n}}, x+\frac{x}{\sqrt{n}}\right], \quad I_{3}=\left[x+\frac{x}{\sqrt{n}}, \infty\right) .
$$

Then

$$
\begin{aligned}
M_{n}\left(g_{x}, x\right)= & \int_{0}^{\infty} g_{x}(t) H_{n}(x, t) d t=\left(\int_{I_{1}}+\int_{I_{2}}+\int_{I_{3}}\right) g_{x}(t) H_{n}(x, t) d t \\
& \stackrel{\text { def }}{=} \Delta_{1, n}(f, x)+\Delta_{2, n}(f, x)+\Delta_{3, n}(f, x) .
\end{aligned}
$$

First, we estimate $\Delta_{2, n}(f, x)$. For $t \in I_{2}$, we have

$$
\left|g_{x}(t)\right|=\left|g_{x}(t)-g_{x}(x)\right| \leqslant \bigvee_{x-x / \sqrt{n}}^{x+x / \sqrt{n}}\left(g_{x}\right)
$$


184

W. Yuankwei and G. Shunsheng

[8]

and so

$$
\left|\Delta_{2, n}(f, x)\right| \leqslant \bigvee_{x-x / \sqrt{n}}^{x+x / \sqrt{n}}\left(g_{x}\right) \int_{x-x / \sqrt{n}}^{x+x / \sqrt{n}} H_{n}(x, t) d t \leqslant \bigvee_{x-x / \sqrt{n}}^{x+x / \sqrt{n}}\left(g_{x}\right)
$$

Secondly, we estimate $\Delta_{1, n}(f, x)$. Let $\lambda_{n}(x, t)=\int_{0}^{t} H_{n}(x, u) d u$. We have

$$
\begin{aligned}
\left|\Delta_{1, n}(f, x)\right| & =\left|\int_{0}^{x-x / \sqrt{n}} g_{x}(t) H_{n}(x, t) d t\right|=\left|\int_{0}^{y} g_{x}(t) H_{n}(x, t) d t\right| \\
& =\left|\int_{0}^{y} g_{x}(t) d_{t} \lambda_{n}(x, t)\right| \\
& =\left|g_{x}(y+) \lambda_{n}(x, y)-\int_{0}^{y} \lambda_{n}(x, t) d_{t} g_{x}(t)\right| \\
& \leqslant \bigvee_{y+}^{x}\left(g_{x}\right) \lambda_{n}(x, y)+\int_{0}^{y} \lambda_{n}(x, t) d_{t}\left(-\bigvee_{t}^{x}\left(g_{x}\right)\right),
\end{aligned}
$$

where

$$
y=x-\frac{x}{\sqrt{n}}
$$

By Lemma 7, we have

$$
\left|\Delta_{1, n}(f, x)\right| \leqslant \bigvee_{y+}^{z}\left(g_{x}\right) \frac{3 x(1+x)}{n(x-y)^{2}}+\frac{3 x(1+x)}{n} \int_{0}^{y} \frac{1}{(x-t)^{2}} d_{t}\left(-\bigvee_{t}^{x}\left(g_{x}\right)\right)
$$

where $n$ is sufficiently large.

Since

$$
\int_{0}^{y} \frac{1}{(x-t)^{3}} d_{t}\left(-\bigvee_{t}^{x}\left(g_{x}\right)\right)=-\frac{\bigvee_{y+}^{x}\left(g_{x}\right)}{(x-y)^{2}}+\frac{\bigvee_{0}^{z}\left(g_{x}\right)}{x^{2}}+2 \int_{0}^{y} \bigvee_{t}^{x}\left(g_{x}\right) \frac{d t}{(x-t)^{3}}
$$

we have

$$
\begin{aligned}
\left|\Delta_{1, n}(f, x)\right| & \leqslant \frac{3 x(1+x)}{n}\left[\bigvee_{y+}^{x}\left(g_{x}\right) /(x-y)^{2}+\int_{0}^{y} \frac{1}{(x-t)^{2}} d_{t}\left(-\bigvee_{t}^{x}\left(g_{x}\right)\right)\right] \\
& \leqslant \frac{3 x(1+x)}{n}\left[\bigvee_{0}^{x}\left(g_{x}\right) / x^{2}+2 \int_{0}^{x-x / \sqrt{n}}{\left.\underset{t}{\bigvee}\left(g_{x}\right) \frac{d t}{(x-t)^{3}}\right] .}^{x}\right]
\end{aligned}
$$

Furthermore, since

$$
\int_{0}^{x-\frac{x}{\sqrt{n}}} \bigvee_{t}^{x}\left(g_{x}\right) \frac{d t}{(x-t)^{2}}+\int_{1}^{n} \bigvee_{x-x / \sqrt{T}}^{x}\left(g_{x}\right) \frac{1}{x^{3} / T^{3 / 2}} \frac{1}{2} x \frac{1}{T^{3 / 2}} d T \leqslant \frac{1}{2 x^{2}} \sum_{k=1}^{n} \bigvee_{x-x / \sqrt{k}}^{x}\left(g_{x}\right)
$$

https://doi.org/10.1017/S0004972700029609 Published online by Cambridge University Press 
it follows that

$$
\left|\Delta_{1, n}(f, x)\right| \leqslant \frac{3(1+x)}{n x}\left[\bigvee_{0}^{x}\left(g_{x}\right)+\sum_{k=1}^{n} \bigvee_{x-x / \sqrt{k}}^{x}\left(g_{x}\right)\right]
$$

Last, we estimate $\Delta_{s, n}(f, x)$. Since

$$
\begin{aligned}
\left|\Delta_{3, n}(f, x)\right| & =\left|\int_{x+x / \sqrt{n}}^{\infty} g_{x}(t) H_{n}(x, t) d t\right| \\
& =\left|\left[\int_{x+x / \sqrt{n}}^{2 x}+\int_{2 x}^{\infty}\right] g_{x}(t) H_{n}(x, t) d t\right| \stackrel{\text { def }}{=} R_{1, n}+R_{2, n},
\end{aligned}
$$

using similar methods as above, we have

$$
\left|R_{1, n}\right| \leqslant \frac{4(1+x)}{n x} \sum_{k=1}^{n} \bigvee_{x}^{x+x / \sqrt{k}}\left(g_{x}\right)
$$

Since $g_{x}(t)=0\left(t^{r}\right) \quad(t \rightarrow \infty)$, there exists $M>0$ such that

$$
\begin{aligned}
\left|R_{2 n}\right| & \leqslant M \int_{2 x}^{\infty} t^{r} H_{n}(x, t) d t \leqslant M \int_{|t-x| \geqslant x} t^{r} H_{n}(x, t) d t \\
& \leqslant \frac{M}{x^{4}} \sum_{k=0}^{\infty}(n-1) \int_{0}^{\infty} P_{n k}(x) P_{n k}(t) t^{r}(t-x)^{4} d t .
\end{aligned}
$$

From

$$
\begin{aligned}
& \frac{(n-1) P_{n k}(t) P_{n k}(x) t^{r}}{(n-r-1) P_{n+r, k}(x) P_{n-r, k+r}(t)} \\
& \quad=(1+x)^{r} \frac{(k+1) \cdots(k+r)(n-1) n \cdots(n+r-1)}{(k+n) \cdots(k+n+r-1)(n-r-1) \cdots(n-1)}
\end{aligned}
$$

it follows that, for every $k$ and $n>r+1$, we have

$$
\frac{(k+1) \cdots(k+r)}{(k+n) \cdots(k+n+r-1)}<1, \quad \lim _{n} \frac{(n-1) \cdots(n+r-1)}{(n-r-1) \cdots(n-1)}=1 .
$$

Hence if $n$ is sufficiently large, then

$$
(n-1) P_{n k}(x) P_{n k}(t) t^{r} \leqslant(1+x)^{r}(n-r-1) P_{n+r, k}(x) P_{n-r, k+r}(t) .
$$


Replacing above in (3.6), we have

$$
\begin{aligned}
\left|R_{2 n}\right| & \leqslant \frac{M}{x^{4}} \sum_{k=0}^{\infty} \int_{0}^{\infty}(1+x)^{r}(n-r+1) P_{n+r, k}(x) P_{n-r, k+r}(t)(t-x)^{4} d t \\
& \leqslant \frac{M}{x^{4}}(1+x)^{r}(n-r+1) \sum_{k=0}^{\infty} P_{n+r, k}(x) \int_{0}^{\infty} P_{n-r, k+r}(t)(t-x)^{4} d t \\
& =\frac{M(1+x)^{r}}{x^{4}} T_{n 4} .
\end{aligned}
$$

Using (2.11), we obtain

$$
\left|R_{2 n}\right| \leqslant \frac{(1+x)^{r}}{x^{4}} O\left(\frac{1}{n^{2}}\right)
$$

From (3.5), (3.6), (3.7) and (3.9), it follows that

$$
\begin{aligned}
\left|M_{n}\left(g_{x}, x\right)\right| \leqslant & \bigvee_{x-x / \sqrt{n}}^{x+x / \sqrt{n}}\left(g_{x}\right)+\frac{3(1+x)}{n x}\left[\bigvee_{0}^{x}\left(g_{x}\right)+\sum_{k=1}^{n} \bigvee_{x-x / \sqrt{k}}^{x}\left(g_{x}\right)\right] \\
& +\frac{4(1+x)}{n x} \sum_{k=1}^{n} \bigvee_{x}^{x+x / \sqrt{k}}\left(g_{x}\right)+\frac{(1+x)^{r}}{x^{4}} O\left(\frac{1}{n^{2}}\right)
\end{aligned}
$$

where $n$ is sufficiently large.

Our theorem now follows from $(3.1),(3.4),(3.10)$; that is

$$
\begin{aligned}
& \left|M_{n}(f, x)-\frac{1}{2}[f(x+)+f(x-)]\right| \\
& \leqslant \frac{50}{\sqrt{n}}\left(\frac{1+x}{x}\right)^{3 / 2}|f(x+)-f(x-)| \\
& \quad+\frac{(1+x)^{r}}{x^{4}} O\left(\frac{1}{n^{2}}\right)+\frac{4(1+x)}{n x} \sum_{k=1}^{n} \bigvee_{x-x / \sqrt{k}}^{x+x / \sqrt{k}}\left(g_{x}\right)+\bigvee_{x-x / \sqrt{n}}^{x+x / \sqrt{n}}\left(g_{x}\right) \\
& \leqslant \\
& \quad \frac{50}{\sqrt{n}}\left(\frac{1+x}{x}\right)^{3 / 2}|f(x+)-f(x-)| \\
& \quad+\frac{5(1+x)}{n x} \sum_{k=1}^{n} \bigvee_{x-x \sqrt{k}}^{x+x / \sqrt{k}}\left(g_{x}\right)+\frac{(1+x)^{r}}{x^{4}} O\left(\frac{1}{n^{2}}\right) .
\end{aligned}
$$




\section{REMARK}

We shall prove that our estimate is essentially the best possible. Consider the function $f(t)=|t-x|(0<x<\infty)$ on $[0, \infty)$. It obviously belongs to $B V_{l o c_{1} r}(0, \infty)$. Since

$$
M_{n}(f, x)=\int_{0}^{\infty} H_{n}(x, t) f(t) d t=\int_{0}^{\infty}(n-1)\left[\sum_{k=0}^{\infty} P_{n k}(x) P_{n k}(t)\right] f(t) d t
$$

by Lemma 6 , for any small $\delta>0$ and $n$ sufficiently large, we have

$$
\begin{aligned}
M_{n}(|t-x|, x) & =(n-1) \sum_{k=0}^{\infty} P_{n k}(x)\left[\int_{x-\delta}^{x+\delta}+\int_{|t-x|>\delta}\right]|t-x| P_{n k}(t) d t \\
& \leqslant \delta+\frac{1}{\delta} M_{n}\left((t-x)^{2}, x\right) \leqslant \delta+\frac{1}{n \delta} 3 x(1+x)
\end{aligned}
$$

and

$$
\begin{aligned}
M_{n}(|t-x|, x) & \geqslant \int_{x-\delta}^{x+\delta}|t-x| H_{n}(x, t) d t \geqslant \frac{1}{\delta} \int_{x-\delta}^{x+\delta}(t-x)^{2} H_{n}(x, t) d t \\
& \geqslant \frac{x(1+x)}{n \delta}-\frac{1}{\delta} \int_{|t-x|>\delta}(t-x)^{2} H_{n}(x, t) d t
\end{aligned}
$$

Furtherfore, using Lemma 6, we have

$$
\int_{|t-x|>\delta}(t-x)^{2} H_{n}(x, t) d t \leqslant \frac{1}{\delta^{2}} M_{n}\left((t-x)^{4}, x\right) \leqslant \frac{c_{1}}{\delta^{2} n^{2}} .
$$

Hence

$$
M_{n}(|t-x|, x) \geqslant \frac{x(1+x)}{n \delta}-\frac{c_{1}}{n^{2} \delta^{2}} .
$$

Choose $\delta=2 \sqrt{c_{1} / n x(1+x)}$. We obtain from (4.1) and (4.2) that

$$
\frac{3[x(1+x)]^{3 / 2}}{8 \sqrt{c_{1} n}} \leqslant M_{n}(|t-x|, x) \leqslant \frac{2\left[c_{1}+(x(1+x))^{2}\right]}{\sqrt{c_{1} n x}} .
$$

For $f(t)=|t-x|$, putting $t=x$, we have

$$
\begin{aligned}
& \left|M_{n}(f, x)-\frac{1}{2}(f(x+)+f(x-))\right| \\
& \quad=\left|M_{n}(|t-x|, x)\right| \leqslant \frac{3(1+x)}{n x} \sum_{k=1}^{n} \bigvee_{x-x / \sqrt{k}}^{x+x / \sqrt{k}}(f)+\frac{(1+x)}{x^{4}} O\left(\frac{1}{n^{2}}\right) .
\end{aligned}
$$


Since

$$
\bigvee_{x-\beta}^{x+\alpha}(f)=\alpha+\beta
$$

it follows that

$$
\begin{aligned}
M_{n}(|t-x|, x) & \leqslant \frac{4(1+x)}{n x} \sum_{k=1}^{n} \bigvee_{x-x / \sqrt{k}}^{x+x / \sqrt{k}}(f) \\
& =\frac{8(1+x)}{n} \sum_{k=1}^{n} \frac{1}{\sqrt{k}} \leqslant \frac{8(1+x)}{\sqrt{n}} .
\end{aligned}
$$

By comparing (4.3) and (4.4), we see that (1.2) cannot be asymptotically improved for $B V_{\text {loc, } r}(0, \infty)$; that is, our estimate is essentially the best possible.

\section{REFERENCES}

[1] A. Sahai and G. Prasad, 'On simultaneous approximation by modified Lupas operators', J. Approx. Theory 45 (1985), 122-128.

[2] R. Bojanic, 'An estimate of the rate of convergence for Fourier series of functions of bounded variation', Publ. Inst. Math. Belgrade (N.S.) 26 (40) (1979), 57-60.

[3] Y.S. Chow and H. Teicher, Probability theory (Springer-Verlag, Heidelberg, Berlin, New York, 1978).

[4] F. Cheng, 'On the rate of convergence of Bernstein polynomials of functions of bounded variation', J. Approx. Theory 39 (1983), 259-274.

\footnotetext{
Department of Mathematics

Hebei Teacher's University

Shijiazhuang

Hebei province

The People's Republic of China
} 